Voix et Images

voixetimages

\title{
La lettre de l'amour et la crise du coeur
}

\section{Louise Desjardins}

Volume 8, numéro 1, automne 1982

Madeleine Gagnon

URI : https://id.erudit.org/iderudit/200363ar

DOI : https://doi.org/10.7202/200363ar

Aller au sommaire du numéro

Éditeur(s)

Les Presses de l'Université du Québec

ISSN

0318-9201 (imprimé)

1705-933X (numérique)

Découvrir la revue

Citer cet article

Desjardins, L. (1982). La lettre de l'amour et la crise du coeur. Voix et Images, 8(1), 35-43. https://doi.org/10.7202/200363ar d'utilisation que vous pouvez consulter en ligne.

https://apropos.erudit.org/fr/usagers/politique-dutilisation/ 


\title{
La lettre de l'amour et la crise du cœur
}

\author{
par Louise Desjardins, Collège de Maisonneuve
}

J'ai lu Au cœur de la lettre dans une sorte de réseau d'adhésions, une sorte de hasard aussi inexplicable qu'une rencontre amoureuse. J'y ai cherché mon étrange identité, attentive aux cris de la vagissante, ma langue maternante qui m'enveloppe et me reconduit au lit, au sommeil de l'enfant. La petite impression de toute la grande vie à vivre.

Je retourne au cœur de la lettre, dans la ligature ancienne des temps stériles, je travaille au cœur des ligaments fibreux et fiévreux, je cherche un col dans le noeud d'origine. La première grille raye la caresse des odeurs. Je suis réduite à l'irréductible catalepsie du désir.

J'écris la lettre de l'amour. Les mots d'amour s'écrivent dans la mémoire de l'amour, vertige amoureux au ralenti. Partir et rester. Seule l'écriture consigne les silences. La parole crue masquerait les replis de furtivité. Les mots du corps se laissent prendre par l'écriture, geste souffle de bouche et de lèvre. Les mots du désir appartiennent à l'écriture du cœur.

Le cœur écrit le rêve du ventre, ni femme ni homme, sans réponse à l'aquestion. L'espace du désir navigue comme il peut entre l'ovaire et le phallus, la fécondité est une affaire entre. Les lettres du cœur parlent erreur, culpabilité, attente, désir qui ne se rend jamais. Quotidien. Transgression. L'innommable est tapi là, derrière le regret. L'indicible révèle les plaisirs dérogeants et dérangeants. Peler la peau, voir si le sang circule chimique entre l'eau et le feu.

L'écriture du cœur est féconde, irréférente, différante. Elle circule l'espace et le temps et le regard sans que la tête ne l'encercle. Empreinte digitale de la passion et de la respiration fondamentale. Le cœur s'écrit et consigne la tendresse de l'absence, trace le contour du corps, le velouté de la peau, le désir à portée d'instant comme une masturbation de l'autre. L'amour écrit le profil de la respiration du même et de l'autre, donne une cadence au risque de la distance.

Le vide écrit la recherche de l'incertain pour découvrir d'autres incertains, entreprise de rêve et d'attente. La ralentie des mots signe la fugacité, temps point, temps ligne. Adagio. La respiration de l'écrit accélère le désir en état de désir. 
Je lis $A u$ cour de la lettre et je meurs à ces mots d'absence et de vide. Forte du fil des mots, je me jette dans le torrent. Ce plus rien. Cette ivresse d'aller quelque part pour aller nulle part.

Je ne prétends rien expliquer de la crise du cœur. Ma lecture veut tout simplement rendre compte d'une rencontre avec ces mots qui m'ont touchée, m'ont parlé angoisse et détresse à travers le plaisir du mouvement partagé.

Je livre également ma déroute dans la fascination, dans l'attirance du dit du non-dit, du vide et de la perte, lecture à la limite de mon endurancejouissance. Je m'apprivoise à l'étendue, à la profondeur, à l'inconnu, au désastre. Aux confins de l'impénétrable et de l'indicible. Beauté. Détruire, dit-elle. Ou l'exigence de l'aventure immolante, la page blanche se promène aller-retour. Je rencontre l'opacité, je me tends de toutes mes forces vers ces lettres gravées, ces lettres de cœur, bien au-delà du cœur en crise.

Mouvement de crise que je ressens comme une blessure qui se refuse. Spasme. Le cœur s'arrête justement pour ne pas s'arrêter. Dans une respiration à la fois emphatique et retenue. La respiration haletante et analgésique de l'effort, de la souffrance à traverser. Projection libérante de la main qui écrit le cri. Je suis (é)mue au fil des pages, impossible d'organiser ma lecture autrement.

D'abord cette phrase de Gauvreau, comme une mise en garde:

Hélas, malheur aux riches? Malheur aux riches du cœur! (p. 59) ${ }^{1}$

Quelque chose d'inattendu se met en branle, le trop est en exergue déjà. II y aura rebondissement ou débordement entre la plénitude et la brisure. «Hélas!» comme un regret crié.

Madeleine Gagnon arrive à la page suivante, marche seule, à la troisième personne «elle». Solennelle.

Il s'agissait d'un lieu étrange où elle se souvient avoir été parce qu'elle avait senti vivement l'extrême normalité des choses. (p. 60)

Une trace émerge d'un état, d'une latence, le mouvement arrivera de très loin et de très fixe et de très mal. Vivement l'extrême brisera et annulera la normalité. Passé futur de l'éclatement.

Ensuite arrive la phrase de Philippe Haeck. Annonce le geste qui s'étire vers le vide:

Le cœur s'use et il. (p. 61)

Un sujet qui n'a de prise sur aucun verbe. Un mouvement qui s'imprime unique et inutile. Un mouvement qui se meut par lui-même. Automoteur.

Dans une formule passive et vague, l'accident rejoint le «je».

Touchée en quelque sorte par de l'accidentel.

J'ai rencontré l'accident, en personne. (p. 61) 
De l'accidentel, la rencontre est partitive, le sentiment devient personne, la virgule permettant de confondre le "je" avec «en personne», contact chavirant et violent qui effacera le «je» jusqu'à la fin de la page, faisant place au «cela» et à l'"ailleurs".

Cela vient d'un ailleurs. (p. 61)

Le "tu» introduit ensuite dans la chair, dans la marque de la chair.

(...) tu n'y étais pour rien et pourtant la chair

de ta chair a été touchée. (p. 61)

Je me dissocie de ce «tu», ce n'est pas moi lisant, je suis moi-même la blessée du vide en nulle part. L'accident arrive en dehors de, blesse le "tu" qui ne comprend pas mais qui percute dans le vide vertigineux du «je». Le lieu de la blessure est «tu» et «je»dans et entre «il», l'accident est «entre».

(...) il était une fois, personne. (p. 62)

Il était une fois virgule personne, volonté de graver une histoire irréférente. Tout se situe au niveau de la représentation garante de l'unicité de la lettre.

Si j'écris mort, si j'écris viol,

si j'écris cri tout seul (p. 62)

Je vois le viol s'écrivant, je vois la mort s'écrivant, tout cela reste suspendu selon la formule de Barthes à propos du cadeau:

Une forme graphique (inscrite dans l'espace de la pièce) est de la sorte donnée à l'acte d'échange en qui, par cette forme, s'annule toute avidité (le cadeau reste suspendu entre deux disparitions). ${ }^{2}$

Le cri tout seul, je ne le vois pas, je l'entends. Il fait une trace dans ma chair, il vient d'une trace qui est trop. Le cri irréférent devient plus puissant que la photo de la faim, il imprime la marque du vide, relais de la trace. Il était une fois, je crie. Il était une fois, tu cries. Il était une fois, il arrive. Le cri, écrit ou vomi, la même vague en crise de soulagement, le coeur ne va plus. II déferle.

Que ceux à qui c'est arrivé l'inscrivent

dans ce point. (p. 63)

Cette phrase me parle et se tait en même temps, elle m'est ouverte comme une porte fermée. Je n'ai pas le goût d'ouvrir cette porte impossible, j'ai plutôt le goût de m'installer devant, bien à l'aise et de rêver. Je fais plusieurs autres points sur la page presque blanche. Cette phrase unique est aussi disponible qu'un haïku. Je contemple et cela devient un sentiment. L'impossible est dessiné dans ce point.

Mes yeux regardent à droite (p. 65) une encre verticale comme l'arbre, comme l'interrogation de Marguerite Duras sur la séparation et la fragmentation. Puis mes yeux reviennent à gauche

(...) étranges arbres le cœur enfoui sous la terre qui gonfle, j'ai peint cet envers (...) j'ai peint le cœur que I'on ne voyait pas, j'ai dessiné l'incoupable, l'innommable. (p. 64) 
L'arbre est fragmenté, son discours est étrange comme lui, discours au troisième degré, trace de la trace. Ce qui est enfoui ne se dit pas, ne s'écrit pas. Cela se peint, se dessine. J'écris que je peins, que je dessine et ce faisant, j'inscris le mouvement qui rue dans ses propres brancards.

(...) d'avoir appris l'inévitable carence, je te dis, dans la démesure violente du rien (p. 64)

Immixtion du cje» et du mouvement, projection du mouvement dans le cri écrit, propulsion auto-protectrice. L'arbre fabrique dans ses racines tout un réseau d'expulsions. Une fois soulagé par l'écrit du cri/le cri de l'écrit, le «je» fait place au ça, au «il y a»

(...) il y a des cris heureux, les cris de toutes naissances (...) ca se donne des airs de fantômes $(. .$.$) on dirait. Tourne la page, cela ne te regarde$ plus. (p. 64)

D'écrire la mort la fait vivre dans l'absence de certitude absolue. Le conditionnel "on dirait" de la naissance par le cri mort-vivant indique un besoin d'atténuer. Je me laisse aller dans le repos factice du doute devant le pire, la porte se referme aussitôt sur la phrase autoritaire qui me claque au nez. Ce «te» de «cela ne te regarde plus» s'adresse peut-être à moi et l'ambiguïté me blesse. Quel est le vrai destinataire de cet impératif? Le vide tout seul est dit, il n'y a plus rien à faire, tout est caché dans le mouvement intérieur automoteur. La vie est là, au-dedans. Imperceptible. Le feuillage apparence de vie vient des apparences de mort.

(...) il ne respire plus mais fait pousser les fleurs, broyer les cailloux et en bas, la rivière continue de couler, rouge des effets du soleil sur les apalaches, chauves, en cet endroit précis. (p. 67)

Les fleurs, seuls témoins des racines enfouies qui coincent l'arbre dans sa boîte qui ne respire plus, mais qui se meut, liquide, à l'intérieur. Idée de labeur, de la mort accouchant de la vie sans accouchement, dans le cri pur et délirant de la vitesse fluide.

II pleut dans cet arbre et je poursuis ma route (p. 67)

Le seul «je" de toute la page embraye immédiatement au «il» impersonnel. L'endroit précis où les apalaches sont chauves me rappelle rouge et vide, l'accident.

Dans le train filant à une allure folle promis à l'affrontement là-bas sur le mur de béton (p. 67)

Le train, boîte motrice vertigineuse, conjonction liquide des axes de longueur (vitesse, file) et de profondeur (arbre, vertige) détermine les conditions de l'accident, l'accident qui survient dans l'axe précisément, affrontement des coordonnées, seule certitude éclatante qui écrasera (béton) durement. Sauve qui peut la vie.

(...) alors certains sautent désespérés (p. 67) 
L'écrit consigne la perte, la vie morte ou presque. La survie survient, l'écrit vient sur, accedere en latin.

(...) d'autres demeurent laissent dans ce tombeau vivant quelques naissances en perte des amours et sur les parois éclatées quelques traces écrites (p. 67)

La page suivante résonne l'orient et me ramène au kaïku, à Barthes.

Ainsi se sont toujours liés, grappes subversives, érotisme et sacré. Des corps se sont sans cesse immolés sur l'autel étal du renoncement. Voilà pourquoi demeure l'écrit, le geste dit le geste peint, nécessaire et en suspens. (p. 68)

Projection étale, cadeau suspendu entre deux disparitions, holocauste de l'écrit désir, volonté de rejoindre ou d'atteindre, érotisme et sacré. Toute offrande est sacrée, tout désir est érotique. Le réseau de la subversion me semble venir d'un ailleurs indéfinissable mais présent.

Arrive une phrase de Marguerite Duras qui dit l'indicible et qui viole ma culpabilité.

Mais il y a un moyen de faire que cette souffrance soit supportable, c'est d'en être l'auteur. (OUTSIDE) (p. 69)

Ambiguïté du mot auteur. Souffrance quotidienne du désir qui n'atteint pas le «tu». Sauf si j'en suis l'initiatrice dans le mouvement ou dans l'écrit. Degré sous zéro dé l'écriture, litote qui projette dehors le tabou de la souffrance "trop», la souffrance outside.

En exergue d'une page qui m'atteint par le cri et le mouvement, vient une citation de Laure, les femmes érotiques n'ont qu'un prénom.

Le scandale du corps parlant tue l'écrivant, amour, comment ressusciter le ciel performatif en mi-décembre et c'est minuit, je t'écris. (p. 70)

Cette phrase est cri pur, la bouche est grande ouverte par les sons qui résonnent dans une grande cavité. "Amour» est respiration dans le cri, puis les sons deviennent stridents, intolérables. Douleur précise qui pointe comme la montagne chauve de l'accident. Interrogation du cri déposé qui veut rejoindre le «tu», fait un appel pressant à la mémoire, le «je» se retire dans le mouvement enclenché par les mains, hors de tout contrôle.

Ce sont mes mains qui les parcourent comme on n'a jamais vu, courir à ce point voler (p. 70)

Le souvenir émerge, trace chaude de juillet. Le temps d'une odeur et se retire aussitôt, sans plus.

(...) toujours en juillet, les autres saisons

confirmant cette odeur, sans plus. (p. 70)

La mémoire revit le regard dans l'interrogation qui fait revenir à son tour le «je», reprend le mouvement qui déplie et enroule, le moi se détache de la boule magma de «je» «tu» «il», file, étoile ourse et interroge le «tu»: 
Je déplie alors les vagues du vent et m'enroule dans la tempête aérienne (...) c'est moi l'ourse tremblante qui parle, le sais-tu? (p. 70)

Le cri semble ensuite se débattre tout seul, angoissant, devient intolérable. Impression de fatigue et d'épuisement. Apaisement par le doute, atténuation.

Comprends que tu me regardais t'aimer immobile et ça s'est terminé comme cela se doit puisqu'un tel cri ne pouvait battre (...) du moins je le crois, sans sa fin. (p. 70)

Après cette projection sidérale, le cri retourne dans l'antre. Mouvement vers l'intérieur, repli dans la crise, spasme de parole de doute, de naissance, de passage, aller-retour du désir et du vide.

(...) je ne sais plus. Les mains imposées pour guérir, et l'aphonique se met à marcher. Un bruit sourd, tu te retournes, de l'antre surgit l'astre figé là (p. 73)

Le «je» du doute met en branle le mouvement de la parole et de la naissance qui vient de la dissociation de «je» et de «elle», le corps se laisse aller, se livre lourdeur et chaleur, porosité dans la réverbération astrale.

(...) on dirait que va s'ouvrir la terre sous mes pieds chauds, que le monde commence puisque j'existe, elle dit. (p. 73)

Le $\propto$ je» revient de nouveau pour constater la présence de «je», constatation qui amorce une sorte de mouvement velléitaire,

(...) je suis. Je donne au mouvement sa parole, je crée le geste. (p. 73)

Et une fois le mouvement imprimé, il s'efface d'un revers de phrase

(...) immobile à voir. (p. 73)

deux fixités contemplatives s'émeuvent alors dans la perte.

(...) offre les miettes d'un délire, où je me retrouve, respire, délie, reçois

l'amour au cœur de la lettre (p. 73)

Le «je" s'étiole de nouveau, on ne sait plus s'il s'agit de l'indicatif ou de l'impératif. Ambiguïté ou coïncidence, l'énigme reste entière, prolonge le chevauchement du «tu» et du «je» dans cette interrogation:

Ta parole donnée maintenant tu la reprends? oui je la reprends, si parfois le vol est nécessaire. II faut avoir beaucoup cru pour n'être plus athée. (p. 73)

L'aphorisme s'étale comme un dogme et annonce la citation de Claudel sur la parole/mort/serment, permet d'entrer parole et serment dans la pensée de Claudel, poète d'une foi.

Je n'apporte qu'une parole... Quelle parole? (...)

La mort n'en fera-t-elle pas autant? 
La métaphore de l'antre revient dans les cinq vers suivants, les seuls de tout le chapitre, comme un besoin d'air, comme un vide respiratoire avant l'effort.

Et j'irai jusqu'à la cage souterraine

Je toucherai de mes mains son cceur de marbre

Je mendierai ton souffle jusqu'à l'extase

Au rythme de mon pouls aphrodisiaque

Je consumerai les noces agoniques (p. 73)

Tous au futur, ces vers marquent la tension du désir vers un autre centre. prison froide, le prix à tout prix qu'il faut payer dans l'absolu d'un désir détruit. Holocauste du désir. Mort. Irréconciliable de la lettre et du corps, le corps à la remorque de la lettre, ne l'atteignant jamais. L'ampleur de la trace est marquée par cette phrase:

Le corps en deçà des mots, toujours. (p. 74)

S'ébauche ensuite une construction énigmatique projetant le désir vers un objet froid et lointain. La dureté «elle» se fusionne en «je» et redevient objet «ce qu'elle». Tension et interrogation vers le «tu»:

Vouloir toucher ta froide Hollande (...) une dureté (...) sais-tu qui elle est, qui je suis, ce qu'elle est (p. 74)

La mémoire part du présent et entreprend une sorte de grand voyage où le passé rejoint trois espaces de bouleversement, lieux notoires de crise en quête de parole et de liberté.

Un jour l'Espagne et la Chine me prirent vivante. Il y eut aussi ce fol amour infini sur les côtes d'Irlande. L'utopie de l'être se défaisait. (p. 74)

Compte à rebours du rêve, l'imparfait "défaisait» vient effacer tout ailleurs dans le temps et dans l'espace, empiète sur le bien-être qui rêve la mère double et étrange, née et parturiente, alternante entre l'Indienne et la Blanche, aléatoire, interrogeante. Bonheur et accident vont se souder avant la question de l'avenir.

Puis une double mère m'aurait enfantée, étrangère à l'autre $(\ldots)$ Indienne ou blanche, née du bonheur et de l'accident. Est-ce que le champ de malheur ou ma terre promise? Ravie, captive d'une fréquence.

(p. 74)

Une sorte d'extase referme le mouvement grand comme l'univers, le ramène dans ses propres battements et pose les prémices d'un accord, d'une accalmie. Phase presque finale de la crise. La mort se place alors quelque part, en dehors du sujet:

La mort prise à témoin? La mort prise à charge, la vie. Je t'abandonne, je te berce, Je te donne tu me reviens. (p. 75)

La mort incommodante oblige, force presque à choisir la vie. Action du «je» sur le «tu» qui finissent par se joindre dans la tendresse du don et de l'abandon. 
Une chair ne peut s'écrire, dans mes enfants, la fuite, je m'écris. (p. 75) Les enfants (chair) et la fuite (je) sont encadrés d'une part par la négation de l'écriture et d'autre part par le «je» s'écrivant, fuite et enfants devenant confondus et dissociés dans l'écriture.

Vient ensuite la résignation enlisante qui accentue la gravité chute des corps dans une pesanteur paralysante. Le «je» vu d'un ailleurs rejoint de nouveau le «tu» dans une interrogation, sorte d'amalgame filé du "je» et du «tu».

Puis un sommeil de plomb (...) il faut bien redescendre en ville, rivée au socle, clouée à la fontaine (...) on me dirait en ces temps-là d'un autre pays (...) collée à mes amours, à quoi donc avais-tu mal? (...) c'est pourquoi nos guérisons te sont impossibles(...) Alors tu te poses la seule question qui te comprenne: à qui donc ai-je eu mal? J'ai mal à l'autre, à mes noces accidentelles. (p. 75)

On est déjà sorti du couple «je»/ «tu», on retourne à l'autre, à «il», à «ses» noces accidentelles, sorte de rencontre fortuite et volontaire de deux axes à la fois réconciliables et irréconciliables.

Puis revient le souffle et je m'y rive (...) et je m'accorde en prévision de cette pause infinie. (p. 75)

L'instant ne s'accorde pas avec l'infini, le «je» reçoit le souffle qui le fait s'arrêter un peu, bouée musicale, respiration douce, le temps d'une échappée vers la longévité. La crise prend fin sur une ouverture pleine d'énergie inscrite dans cette phrase de René Char:

Il faut mettre en liberté tous les instants qui nous restent. (p. 76)

Et me fait penser à cette autre phrase de Deleuze et Guattari:

C'est par perte qu'on progresse et qu'on prend de la vitesse. ${ }^{3}$

Fermeture de crise, spasmes qui ouvrent sur l'inconnu, rupture nécessaire à l'avènement. L'accident est arrivé sur le sujet, l'a atteint dans un temps précis et imprévisible, dans un lieu précis et imprévu.

$(\ldots)$ on ne sait ou on ne sait pas encore on ne saura pas avant la fin I'on sait seulement l'accident (p. 67)

Le mouvement percute dans la crise, repli sur moi, projection libérante, cri/ouverture, cri/outside, cri/éclat de la trace, cri écrit. Comme un cadeau suspendu. Des lignes vertigineuses se sont rencontrées, se sont traversées, point d'effraction, ont continué dans la perte de ce contact brutal. Continuent.

11 pleut dans cet arbre et je poursuis ma route. (p. 67)

Je referme le livre et je continue de me faire bercer par ce mouvement. Flux et reflux, je reste sur la plage à regarder fluide le mouvement autonome de la mer.

Je n'ai pu entrer dans la vague, repoussée chaque fois par le ressac. L'ouverture éblouissait, toujours fragile, l'anfractuosité du leurre. 
Je clos ce chapitre de crise, fatiguée d'avoir frôlé, épuisée par la dansecontact des yeux et du cour. Tous les mouvements m'ont parlé, m'ont portée, circulaire, linéaire, spatiale, temporelle, intérieure, extérieure, enveloppée. Repli, retour, aller, percussion, la fuite étoile filante et fixe. L'arrêt, l'embrayage, la vitesse, l'éclat. La rencontre, le désir.

Et je suis restée telle la sceur blessée, Ariane, aux bords où je fus laissée. Un peu morte, un peu abandonnée. Dans une sorte de désir indéfinissable. $\mathrm{Im} /$ possible. Et je continue de chercher une petite fille qui se promène seule dans les rues d'Abitibi. Cette écriture comme un miroir de rêve éveillé endormi vécu me lit droit au cœur.

1. Les citations suivies d'un numéro de page renvoient toutes à $A u$ caur de la lettre, Madeleine Gagnon, VLB éditeur, 1981.

2. Roland Barthes, L'empire des signes, Flammarion, 1970, p. 87.

3. G. Deleuze et F. Guattari, Mille plateaux, Minuit, 1980, p. 64. 